\section{Diakonie unter Spardruck ${ }^{1}$}

\section{Lukas Kundert}

Am ersten Advent 2014 fiel der Startschuss für die neue Glaubenskampagne, die in Basel während des darauf folgenden Kirchenjahres durchgeführt wurde.

Wir hatten erkannt, dass es nicht gut ist, in der Öffentlichkeit nur von unserem diakonischen Handeln zu sprechen. Wir haben aber erkannt, dass wir auch den Inhalt, wieso wir diakonisch wirken, in die Öffentlichkeit tragen müssen.

Ansonsten entsteht der Eindruck, wir würden uns des Evangeliums schämen («Wir sind zwar Kirche, aber wir tun auch Gutes»). Entscheidend ist, dass wir Kirche sind - und deswegen, weil wir von einer starken Kraft getragen werden, tun wir auch Gutes.

2008 haben wir mit «best bible stories» überall im Kanton die besten Bibelgeschichten in einer ungewohnten und frech modernen Art aufgeführt. Dann gaben wir der Bevölkerung Gebetssprache - ein Basler Gebetbuch von unterschiedlichen prominenten und unbekannten Stadtbaslerinnen und Stadtbaslern, Zeitgenossen und Vorfahren. Denn die Gebetssprache geht bei uns verloren. Dann boten wir Glaubenskurse an, und während eines ganzen Jahres kurvte die Kirchentram über das Schiennennetz der Basler Verkehrsbetriebe und der Baselland Transport.

Später hat der Kirchenrat dann einen Katechismus erlassen. Da steht nicht drin, was Reformierte glauben müssen, sondern was der Kirchenrat glaubt. Der Kirchenrat ist also mit einem Bekenntnis in die Öffentlichkeit getreten und gesagt hat: Wir glauben.

1 Referat gehalten an der Diakoniekonferenz vom 11. November 2014 in Bern.
Die Vernehmlassung dazu hat keinen einzigen theologischen Vorbehalt gegen diesen Katechismus ergeben, höchstens die Sprache, den Stil der direkten Rede oder einzelne Metaphern betreffend ist es zu kritischen Reaktionen gekommen.

Im Jahr 2014 startete eine neue Kampagne mit dem Titel «feste feiern». Beide Wörter sind kleingeschrieben. So kann «feste» gelesen werden als Substantiv und als Adverb - «Feste feiern» und «feste feiern».

Jede Gemeinde hat ihre Spezialitäten zu einzelnen kirchlichen Festtagen entwickelt. Diese wurden im Verlauf des darauf folgenden Jahres ins Schaufenster gestellt.

Die Idee dahinter: Man weiss, dass das Gefühl der Beschleunigung sich objektiv nicht erhärten lässt. Es hängt damit zusammen, dass die Menschen verlernt haben, die Zeit zu takten: Die Nacht wird zum Tag, im Dezember essen wir Erdbeeren, im Januar Spargeln. Wir begehen die Feste nicht mehr als Jahresfeste und haben verlernt, die Tageszeiten zu begehen. Man empfiehlt, sich an den Angeboten, die Zeit zu takten, der Religionen zu orientieren. Weil man vergisst, was die Feste sind und was sie bedeuten, stellen wir sie nun gross aus und bringen ihren Sinn unter die Bevölkerung.

Parallel zu den Kirchgemeinden veranstaltet die Kantonalkirche im Münster sogenannte Leuchtturmveranstaltungen, die die mediale Aufmerksamkeit auf sich ziehen können. Am ersten Advent predigt Margot Kässmann über den Sinn der Feste. Am Weihnachtsabend wird im Gottesdienst die erste Kantate des Weihnachtsoratoriums mit Knabenkantorei und Orchester aufgeführt. An Karfreitag ziehen wir mit dem Kreuz und der Offenen Kirche durch die ganze Stadt zu Kreuzesorten. Am Ostersonntag verwirklichen wir das Weltwunder der Hängenden Gärten der Siramis im Münster: Wir hängen in das Münster einen Garten. Denn der Auferstandene erschien Maria Magdalena im Garten und sie meinte, er sei der Gärtner. An Pfingsten lassen wir die Weltsprache erklingen: Eine Nacht der Musik, christliche Jazz- und Bluesmusiker aus aller Welt spielen auf. Am Dank-, Buss- und Bettag stellen wir auf den Münsterplatz einen riesigen langen Tisch, an dem 900 Personen Platz finden. Wir
Jahrbuch Diakonie Schweiz 1 (2017) - ISSN 2504-3994

Dieser Text ist lizenziert unter einer Creative Commons Namensnennung 4.0 International Lizenz (CC BY 4.0): (https://creativecommons.org/licenses/by/4.0/).
Jahrbuch Diakonie Schweiz 1 (2017) http://dx.doi.org/10.22018/JDS.2017.6 
laden die ganze Stadt, Juden, Christen und Muslime und Bahais und Buddhisten zum Agape-Mahl auf den schönsten Platz Europas. Und am Reformationsfest bringen wir die Stadt in Form von Porträts von ganz vielen unserer Bevölkerung in das Münster - denn dann feiern wir, dass $\mathrm{Du}$ - auch $\mathrm{Du}$ - von Gott direkt angesehen bist.

\section{Was gibt es denn zu feiern?}

Aber was gibt es denn zu feiern? Sind wir in Basel denn nicht diejenigen, die am meisten von Mitgliederrückgang und Restrukturierungen gebeutelt sind? Haben wir denn wirklich zu feiern und Gott zu danken?

Ja. Das Bild, das in der Schweiz von der Basler Kirche gemalt wird, teilen wir nämlich ganz und gar nicht. Wir haben erkannt, dass uns Gott eine Aufgabe zugedacht hat, die segensreich ist und in der er etwas mit uns und der Kirche vor hat. Wir sind mitnichten eine Kirche in Finanznot. Wir stehen sehr gut da. Wir gehören zu den grössten Zahlern im SEK. Denn wir haben unsere Aufgaben gemacht, was in der Tat auch sehr schwierig und sehr schmerzhaft war. Wir haben es aber im Vertrauen getan, dass nicht wir uns tragen, sondern Gott uns trägt und wir von ihm so unendlich viel Kraft und Charisma geschenkt erhalten - wir müssen ihm nur Raum geben.

Ich darf nicht verhehlen, dass es natürlich auch in unserer Kirche die Mühsamen und Beladenen gibt. Auch bei uns gibt es die, die nur den Verlust sehen und nicht den Reichtum. Wir haben sie zu tragen. Wir haben sie wie Pferdchen an die Hürden zu führen und ihnen zu zeigen, dass man sie überspringen kann. Aber springen müssen sie selbst. Und wenn sie es nicht schaffen, dann lassen wir sie selbstverständlich nicht stehen. Aber wir lassen uns von ihnen auch nicht herunterziehen.

Ich darf Ihnen davon erzählen, wie wir den Turnaround schaffen.

\section{Drei Säulen: Verkündigung, Gemeinschaft und Diakonie}

Die Handlungsfelder der Kirche stehen auf drei Säulen: Verkündigung, Gemeinschaft und Diakonie. Diese drei verhalten sich zueinander fast wie die Trinität: Verkündigung ohne Gemeinschaft gibt es nicht, ebenso wie Gemeinschaft ohne Diakonie, Diakonie ohne Verkündigung und es Verkündigung ohne Diakonie nicht geben kann.

Verkündigung, Gemeinschaft und Diakonie sind Querschnittsfunktionen der Kirche. Sie kommen in allem ihrem Handeln vor. Verkündigung ist nicht alleine die Predigt des Pfarrers, sondern auch das lebendige Zeugnis des BASF-Mitarbeiters in seiner Arbeitsgruppe. Diakonie ist nicht nur das professionelle soziale Handeln des Sozialarbeiters, sondern auch die Beratung der längst pensionierten Margrit für die längst pensionierte Hedi. Und Gemeinschaft ist nicht nur das Kinderlager, das Abendmahl oder die Alterswoche, sondern auch die Seelsorge, die auch Verkündigung und auch Diakonie ist, sie ist auch der Brief aus den Ferien oder der Bibelgruss, den wir einander schenken und vieles mehr. Sie ist auch die Mission, die wir vor Ort, in unseren Gemeinden leisten, und in Übersee, in eigenen kirchgemeindlichen Missionswerken und in unserem grossen Missionswerk von mission21, dort, wo wir Reformierte Weltkirche sind.

Aber wie schaffen wir es, dass dies auch tragend wird?

\section{Die Arbeit ist zu vie}

Im Herbst 2014 hat ref.ch ein mehrteiliges Interview mit einer jungen Pfarrerin und zwei jungen Pfarrern veröffentlicht, die je in einem Einzelpfarramt sind oder waren. Bigna Hauser war die einzige Pfarrerin unter den drei Pfarrpersonen. Sie hatte vor einiger Zeit ihr Einzelpfarramt in den Bündner Bergen aufgegeben und den Beruf gewechselt. Einige ihrer Aussagen haben mich sehr beeindruckt, und ich will von ihren Aussagen ausgehen, wenn ich über die überlebensnotwendige Restrukturierungen in unseren Kirchen schreibe. 
Frau Hauser wurde gefragt, ob denn die Arbeit im Einzelpfarramt nicht einfach taff sei. Darauf antwortete sie: «Taff finde ich das falsche Wort. Es suggeriert, dass die Anforderungen gerade so liegen, dass sie eigentlich noch cool und fordernd sind. Das stimmt aber nicht. Die Arbeit ist nicht einfach taff, sie ist einfach zu viel.» Diese Antwort ist so wunderbar direkt und ehrlich, dass sie unbedingt gehört werden soll. «Die Arbeit ist nicht einfach taff, sie ist einfach zu viel.» Wir wissen, dass solche ehrlichen Aussagen überhört werden. Man will sie nicht wahrhaben und personalisiert die Probleme. Man macht in der Kirche strukturelle Probleme zu persönlichen Versagen. Diakone und Gemeindeglieder maulen über die Pfarrerin, der Pfarrer über die Diakonin, alle maulen über den Kirchen- oder Synodalrat. Sie seien schuld an der Misere.

Dieses Personalisieren der Probleme hängt daran, dass in unseren Kirchen ein positivistisches Verständnis von Landeskirche vorherrscht. Volkskirche ist in diesem Verständnis immer das Bestehende. Im positivistischen Verständnis von Volkskirche sind es die Werte, die Moral und die Moralinstitutionen, die die Kirche zur Volkskirche machen. Dementsprechend wirbt man für die Kirche als einer Wertegemeinschaft oder als eine Gemeinschaft, die sozial handelt. Das sind moralistische (gesetzliche) Kirchenverständnisse. Sie sind nicht theologisch.

Ich habe den Eindruck, dass in unseren Kirchen, ihren Leitungen, Pfarrpersonen und in vielen ihrer Mitglieder ein solches positivistisches Verständnis von Volkskirche vorherrscht, und man ist dabei der Meinung, dass es sich dabei um ein aufgeschlossenes und modernes volkskirchliches Verständnis handelt. Man verkennt, wie moralistisch und veränderungsfeindlich dieses Modell ist. Denn einem positivistischen Verständnis der Volkskirche ist nur der vergangene Wandel positiv integriert, die aktuelle oder zukünftige Erneuerung wird aber als Veränderung der Volkskirche zugleich als ihre höchste Bedrohung wahrgenommen. Deswegen sind Volkskirchen erneuerungsresistent: Wer eine «CityKirche» gründet oder eine «Stadtakademie», muss es gegen sehr viel Widerstand tun und wird, wenn sie einmal bestehen, sie nie mehr auflösen können. Innovationen werden also doppelt bestraft: Bei ihrem Aufbau und bei ihrer Streichung. Mit einem Wort des ehemaligen Ratsvorsitzenden der EKD, Bischof Wolfgang Huber, gesagt: «Volkskirche, ursprüng lich ein Reformbegriff, wirkt legitimierend für den Status quo.»

Wir können an ganz unterschiedlichen Ecken anfangen und die grundsätzliche Krise unserer Kirchen konstatieren. Wir können den Mitgliederrückgang werten und uns darauf einstellen, dass wir finanziell einige Abstriche machen müssen. Aber wir stehen nicht finanziell mit dem Rücken zur Wand. Es geht doch fast allen finanziell hervorragend. Wir stehen aber in anderer Weise als Kirche mit dem Rücken zur Wand. Wir könnten nämlich auch den Umstand bedenken, dass es uns nich gelingt, die Massen unserer Gesellschaft zu erreichen und mit dem, was in uns brennt und uns Alles ist, zu begeistern. Lediglich ein relativ kleiner Teil unserer Mitglieder ist mit der Botschaft, die in uns brennt, zu erreichen. Wir können auch bedenken, wieso es für viele beschämend geworden ist, sich als Christinnen und Christen zu outen. Wir können bedenken, weswegen es an unseren Fakultäten nicht mehr gelingt, eine aktuelle Theologie zu entfalten und stattdessen Selbsthistorisierung betrieben wird. Oder wir können einfach von der Aussage von Frau Hauser ausgehen: «Die Arbeit ist einfach zu viel.» Ich ziehe es vor, diese Aussage als Ausgangspunkt zu nehmen. Denn es geht mir in diesem Referat nicht einfach nur um die Kirche oder um die Gemeinde, sondern es geht mir insbesondere u darum, dass alle Beteiligten sich ein Umfeld erkämpfen können, in dem sie nicht nur gesund bleiben, sondern in dem sie eine lebendige und alle mittragende Gemeinde aufbauen helfen können.

Ich komme nochmals auf Frau Hauser zu sprechen. Sie sagt an einer anderen Stelle im Interview: «Man ist ständig daran, alle Bälle, die in der Luft sind, im Blick zu haben und zu jonglieren. Das zusätzliche Problem dabei ist eben: Jeder Ball hat nur Kenntnis von sich und nicht von den Dutzenden anderen Bällen, die auch noch in der Luft schweben. Ich glaube, viele Senioren haben keine Ahnung davon, was in der Kinderarbeit so läuft und sie interessiert es auch nicht, ob man noch eine zusätzliche Klasse in Religion unterrichtet. Die Senioren wissen aber sehr genau, bei wem man zu Besuch war - und bei wem eben nicht.» 
Das Bild der Artistin in der Manege, die permanent Bälle in der Luft und in Bewegung halten muss, ist mir nachgegangen. Ich meine, zu verstehen, was Frau Hauser hier meint. Und ich will das Zitat aber etwas dehnen. Ich meine, dass die Pfarrerin oder der Pfarrer und auch die Diakoninnen und Diakone nicht nur die Bälle in Bewegung zu halten und zu steuern haben, sondern dass sie zugleich noch in jedem Ball präsent sein müssen: Im Ball Krabbelgottesdienst ebenso, wie im Ball Sonntagsschule; im Ball Religionsunterricht ebenso, wie im Ball Jugendgruppe; im Ball Männergesprächskreis ebenso, wie in der Golden Agers Gruppe und so weiter: Gebetsgruppe, Frauenkreis, Israelkreis, HEKS-Gruppe, Missionsgruppe... Der Aktivitäten sind Legion. Und in jede springen wir hinein. Wir jonglieren die Bälle und springen in die Bälle hinein. Ich habe noch keine Artistin gesehen, die das beherrscht. Wieso nur trauen wir uns das zu?

\section{Ein heilloses Dogma: Die Pro-Kopf-Finanzen}

Ich will hier zuerst vom Geld sprechen. Die Volkskirchen im deutschsprachigen Raum kennen ein ganz und gar unbiblisches Allokationsdogma. Jede Gemeinde soll im Verhältnis zu ihrer Mitgliederzahl Geld erhalten. Faktisch bedeutet das, dass es ab 2000 Gemeindegliedern eine volle Pfarrstelle geben darf. Es ist dabei völlig egal, wie sehr sich diese 2000 Gemeindeglieder kirchlich engagieren, was für Gruppen es gibt und wie lebendig diese Gruppen sind. Verlangt wird nicht, dass und in welcher Weise diese Gemeinde lebt, sondern dass es die Köpfe gibt.

Eine Person, die in einer solchen Struktur Verantwortung übernimmt, wird sehr viel Engagement bringen, aber nie für das Engagement «belohnt» werden. Im Gegenteil. Angesichts der demographischen Struktur wird sie Jahr für Jahr ausgesprochen oder unausgesprochen den Vorwurf hören, nicht zu genügen. Denn es werden weniger Mitglieder eingeschrieben sein (ausser man lebt in den Kantonen Schwyz oder Freiburg, wo die Gemeinden wegen Zuzug etwas wachsen).
Das Dogma der Pro-Kopf-Finanzen ist radikal zu brechen. Denn es handelt sich bei diesem Dogma um eine Fessel, die Innovationen nicht belohnt, sondern alle Kraft auf das Verwalten des Status Quo fokussiert. Dieses Dogma nimmt auch nicht zur Kenntnis, dass eine Person maximal 300 Personen «binden» kann. Die staatliche Sozialarbeit beschränkt die Anzahl Dossiers, die eine Sozialarbeiterin zu betreuen hat, auf 120 Stk; ein Aussendienstmitarbeiter einer Versicherung hat höchstens 200 bis 300 Dossiers zu betreuen. Nur die Kirchen gehen noch davon aus, dass ein Pfarrer oder eine Pfarrerin wie noch vor 100 Jahren 2000 Seelen betreuen kann und dabei, wenn es gut geht, vielleicht noch eine Diakonin oder einen Diakon zur Seite gestellt erhält.

Stellen Sie sich vor, was Sie alles leisten müssten, um in einer 2000Seelen-Gemeinde einen Gottesdienstbesuch von 500 Gottesdienstteilnehmern zu erreichen! Wenn Sie maximal 300 Personen erreichen können, so müssten Sie sich selbst verdoppeln. Aber es gelingt den meisten ja nicht einmal, 300 Personen zu erreichen. Denn die tatsächlich erreichbare Zahl ist für uns Durchschnittsmenschen, die wir auch sein dürfen, eben viel tiefer - wohl bei 100 bis 200 Personen.

Doch Volkskirchen gehen selbstverständlich davon aus, dass sie nur 10 Prozent ihrer Mitglieder ansprechen können. In Basel zählte man 1970 140`000 Mitglieder; aber für diese Mitglieder bot man lediglich 14`000 Sitzplätze in den Kirchen an. Man ging also selbstverständlich davon aus, dass auch an den hohen Feiertagen maximal 10 Prozent der Gemeindeglieder die Kirche aufsuchen wollen. Oder mit anderen Worten: Man ging davon aus, dass 100 Prozent der Kirchenmitglieder für die Kosten aufkommen, die 10 Prozent der Kirchenmitglieder generieren.

\section{Der erste Schritt: Wir brechen das Allokationsdogma}

Die erste Arbeit, die Kirchenleitungen (und damit auch Pfarrkapitel und Synoden) leisten müssen, ist, den Kirchenaufbau in die eigenen Hände zu nehmen und den aus dem Beamtentum übernommenen Automatis- 
mus der Geldzuteilung zu brechen. Denn so, wie die Gelder der Kirche gelenkt werden, so wird sich die Kirche entwickeln. Das bedeutet:

- Die Synode hat unter inhaltlichen Vorgaben zu bestimmen, welche Gemeinden unter welchen Umständen welche Mittel erhalten.

- Die Synode hat einerseits Charismen zu fördern und andererseits dort einzugreifen, wo Unnötiges gemacht wird oder wo die falschen Leute auf die richtigen Aufgaben angesetzt sind.

- Alle Gemeinden sollen einen Minimalstandard sicherstellen (Taufe, Sonntagsschule, Religionsunterricht, Konfirmationsunterricht, Sonntagsgottesdienste, Erwachsenenarbeit, Altersarbeit Seelsorge, Diakonie, Kasualien), aber nicht alle sollen darüber hinaus alles machen, was die anderen auch anbieten (Theaterkirche, Konzertkirche, Jugendkirche, Gospelkirche, gottlose Kirche, fromme Kirche) und vor allem sollen nicht alle alles gleich machen.

- Die vielgelobte theologische Vielfalt in der reformierten Kirche ist faktisch leider eine theologische Monokultur. Jedenfalls wird es Aussenstehenden schwer fallen, die feinen theologischen Unterschiede zwischen Linksbarthianern und Links-Ebelingianern zu erkennen. Wir haben deswegen theologische Differenzen zu fördern und auszuhalten - und in versöhnter Verschiedenheit zu leben.

Alle Gemeinden und Dienste der Kirche müssem eine SWOT-Analyse durchlaufen. Darunter geht es nicht. Schon in der Art, wie diese Analyse erfolgt, zeigt sich, ob eine Gemeinde überlebensfähig ist oder sich selbst Sand in die Augen streut.

\section{Der zweite Schritt: Wir fördern Innovationen}

Die Volkskirchen sind - ich habe es schon gesagt - erneuerungsresistent. Dies kann nur dann gebrochen werden, wenn Innovationen belohnt und gefördert werden. Mindestens 5\% des gesamten Budgets von Gemein- den und Kantonalkirche muss für Innovationen bereitgestellt werden. Dieses Geld darf nicht im Sinn von Projektmitteln auf einen begrenzten Zeitraum (von etwa 4 bi 6 Jahren) beschränkt sein, sondern es soll so lange fliessen, wie die vorab geklärten Parameter erfüllt werden.

Ein Beispiel: Die reformierten Kirchen haben im 19. Jahrhundert die Basler Mission und nach dem Zweiten Weltkrieg das HEKS gegründet und dann später noch das Brot für alle. Das sind Organisationen, die zum Teil höchst innovativ geblieben sind (wie die Mission), die sich aber zum Teil auch überlebt haben. Was gibt es aber an neuen diakonischen Werken? Gibt es wirklich noch Orte, an denen Kirche das leistet, was nicht auch weltliche Hilfswerke leisten?

Im Anspruch, etwas Neues zu kreieren, das nur Kirche kreieren kann, setzen wir uns in Basel zum Ziel, alle zehn Jahre ein neues diakonisches Werk zu gründen. So entstand die Idee vom Sonntagszimmer. Wir haben in Basel eine beachtliche Anzahl von Stadtklöstern und Kommunitäten, und wir haben grosse, gepflegte, wertvolle und gut ausgerüstete Gemeinschaftsräume, und wir verfügen über ein Netzwerk von Menschen, die in unterschiedlichsten Berufen arbeiten und Kompetenzen mitbringen.

Andererseits gibt es ein Quartier, das Untere Kleinbasel, in dem kaum noch Reformierte leben, dafür aber sehr viele grosse meist alawitische Familien. Es ist ein Quartier, in dem es fast ausschliesslich 2- und 3Zimmerwohnungen gibt. Was tun diese Familien am Sonntag?

Unsere Idee: Wir öffnen die Matthäuskirche «von Acht bis Nacht», wir bieten selbstverständlich Gottesdienste und Gebetszeiten an, aber nicht nur: Es gibt an sorgfältig gedeckten Tischen Frühstück, Mittagessen und Abendessen. Es gibt Spiele und Unterhaltung für Jung und Alt. Es gibt Gesang und es gibt Psychotherapie und andere Beratung. Es sind das alles unsere Mitglieder, die sich hier engagieren, und zwar gratis. Ein schönes Projekt. 


\section{Der dritte Schritt: Wir fördern Selbständigkeit und Eigen-} verantwortung

Die Volkskirche belohnt heute Menschen, die den Status Quo erhalten. Sie stellt jenen Menschen, denen der Status Quo nicht reicht, keine Unterstützung zur Verfügung. Das ist unfair. Deswegen haben wir die Möglichkeit geschaffen, dass jede Gemeinde und jeder Gottesdienstort (und das könnten auch die kantonalkirchlichen Ämter tun) eigene zusätzliche Stellen aufbauen und finanzieren können. Der einfachste Weg dazu ist:

- Erarbeiten eines Konzepts; z.B. Jugendarbeit, Mittagstisch, Hauskreisarbeit...

- Gründung eines Fördervereins

- Einwerben der Mittel

- Go for it

\section{Der vierte Schritt: Die jonglierende Pfarrerin}

Eine Pfarrerin oder ein Pfarrer, die in dieser Weise Gemeindeaufbau betreiben kann, wird sich aus vielen ihrer angestammten Tätigkeitsfelder schrittweise zurückziehen können. Sie wird viel von dem, was dem Pfarrberuf in den 1960er bis 1990er-Jahren zugewachsen ist, wieder abgeben können. Sie wird viel mehr Diakone benötigen. Nicht einfach Sozialarbeiter, sondern Diakone. Ideal ist, wenn eine Pfarrerin sich auf den Sonntagsgottesdienst konzentriert, auf die Konfirmandenarbeit und allenfalls noch auf Kasualien (aber auch da nicht alle!). Der Rest ihrer Arbeit soll darin bestehen, die Leiterinnen und Leiter der einzelnen Gruppen der Kirchgemeinde anzuleiten, sie zu führen und für sie neue Ideen zu entwickeln. Nur in einer solchen Struktur wird man nicht permanent aus dem Mangel schöpfen müssen, sondern wird die Musse haben, Fülle entstehen zu lassen.

Ich selbst kenne den Alltag einer volkskirchlichen Situation aus der Pfarrerperspektive. Der Durchschnitt von 70 Stunden Arbeit pro Woche ist mir bekannt. Und es ist mir bekannt, dass das von Zeit zu Zeit viel- leicht so sein muss, aber dass es, wenn es anhaltend so zu und her geht, mich innerlich zerstört, wenn ich so viel für die Kirche unterwegs sein muss. Wir leben in einer Kirche, die aber solche Selbstausnützung fördert und fordert. Wer «nur» 50 Stunden arbeitet, gilt schnell als faul. Dabei sollten diese Stunden doch genügen. Sie können aber nur dann genügen, wenn es gelingt, dass die Pfarrerin oder der Pfarrer nicht, in allen Bällen, die sie jonglieren immer auch zugleich präsent sein müssen. Dazu muss das Ziel sein, dass in der Kirchgemeinde die Pro-KopfBetreuungszahl von 2000 Personen/Mitarbeitender schrittweise reduziert wird auf höchstens 300 Personen/Mitarbeitender. Das muss auch auf dem Land möglich sein.

Ich rate davon ab, in einer Kirchgemeinde den Dienst anzutreten, die nicht dazu Hand bietet, an solch einem Gemeindeaufbaumodell mitzuarbeiten.

\section{Wie sieht das konkret aus? - Das Beispiel der ERK BS}

Ich führte das Beispiel der Münstergemeinde an, also einer Innenstadtgemeinde, die in den vergangenen Jahren drei Viertel ihrer Mitglieder verloren hat. Ich zeige Ihnen dieses Beispiel, weil es zeigt, was alles möglich ist und was Gott alles möglich macht. Aber es ist kein Sonderfall. In anderen Gemeinden ist man sogar noch weiter oder ähnlich unterwegs. Sie sehen hier, wie die Münstergemeinde, die sich nach volkskirchlichen Verhältnissen eigentlich höchstens zwei Pfarrstellen und vielleicht eine Diakonin oder einen Diakon leisten könnte, sich einen weiten Mitarbeiterstab aufgebaut hat, ohne auf den Segen der Steuermittel zu warten.

Es ist so viel möglich. Es braucht dazu viel Mut, Phantasie und vor allem Gottvertrauen. Alles das wünsche ich Ihnen herzlich.

Autor:

Lukas Kundert

Pfr. Prof. Dr., Kirchenratspräsident der Evangelisch-reformierten Kirche BaselStadt 